\title{
Patients' understanding on the aim and duration of treatment during the consent process
}

\author{
Authors: Muhammad Bilal Razzaq, ${ }^{A}$ Konstantinos Kamposioras, ${ }^{B}$ Sarah Corker, ${ }^{C}$ Frances Fox, ${ }^{D}$ George Papaxoinis, ${ }^{E}$ \\ Jane Murray, ${ }^{\mathrm{D}}$ Ruth Peters, ${ }^{\mathrm{C}}$ Janice Padgett, ${ }^{\mathrm{C}}{ }$ Yvonne Brookes, ${ }^{\mathrm{C}}$ Liz Mcguire, ${ }^{\mathrm{C}}$ Laura Lodge ${ }^{\mathrm{C}}$ and Gireesh Kumaran ${ }^{\mathrm{F}}$
}

\section{Background}

Patients starting chemotherapy have to process a significant amount of information about their diagnosis and treatment. In this audit, patients were interrogated regarding the value of information they received before consenting for their treatment, and how this information contributed in their actual understanding of treatment purpose and duration.

\section{Materials}

A standardised questionnaire was used to obtain patients' feedback about the quality of information they had during the consent process, with specific questions about the aim and duration of treatment. The feedback from the questionnaire was compared independently by two clinicians to the information on case notes.

\section{Results}

Sixty-five patients ( 42 males) with a mean age of 65 years were assessed, $32(49 \%)$ with lower- and $33(51 \%)$ with upper-gastrointestinal (GI) malignancies. Of them, 36 (55\%) had palliative, $22(34 \%)$ adjuvant and $7(11 \%)$ neoadjuvant chemotherapy.

More than $90 \%$ of patients stated that the quality of information about their cancer (name, location and spread in the body; medications; side effects and goals of treatment) was excellent, good or satisfactory. Less than $7 \%$ rated the quality as poor. Also, $51(79 \%)$ patients stated that they had appropriate information according to their expectations, while $91 \%$ had the opportunity to ask questions. Nevertheless, one in five patients found it difficult to understand the information.

Based on physicians' assessment, $19 \%$ of the patients did not understand the aim and $29 \%$ did not appreciate the

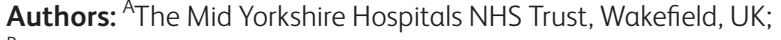
${ }^{B}$ medical oncology consultant, The Mid Yorkshire Hospitals NHS Trust, Wakefield, UK; ${ }^{C}$ chemotherapy sister, The Mid Yorkshire

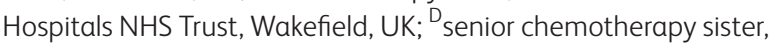
The Mid Yorkshire Hospitals NHS Trust, Wakefield, UK; Emedical oncology consultant, second department of oncology, Agios Savvas Anticancer Hospital, Athens, Greece; F medical oncology consultant, The Clatterbridge Cancer Centre NHS Foundation Trust, Wirral, UK
}

duration of the treatment. Only one-third of the patients on adjuvant treatment understood both the aim and the duration of the treatment. Males compared with females ( $81 \%$ vs $52 \%$, respectively; $p=0.018$ in multivariate analysis) and those on palliative or neoadjuvant compared with those on adjuvant treatment $(80.6 \%$ or $85.7 \%$ vs $50 \%$, respectively; $p=0.03$ in univariate analysis) seemed to better understand the duration of treatment.

\section{Conclusion}

Patients consenting for chemotherapy are overwhelmed with a plethora of information. Patient understanding and choice is important especially in the adjuvant setting as a significant proportion would not benefit from the treatment with high likelihood of side effects during the treatment and up to $20 \%$ chance of long-term and disabling toxicity.

\section{Conflict of interest statement}

None declared. 